\title{
Robust predictive Control for Networked Control Systems with Access Constraints
}

\author{
Pan Zhang \\ College of Electrical and Information Engineering, Lanzhou University of Technology, Lanzhou \\ 730050, China \\ 2280980151@qq.com
}

Keywords: Networked control system, medium access constraints, packet loss, robust predictive controller

\begin{abstract}
Based on the rolling horizon optimization strategy, the networked robust predictive control with medium access constraints and packet loss is studied. Firstly, considering the influence of network factors such as medium access constraints and packet loss, Markov jump rule and Bernoulli independent and identically distributed process are used to transform the network problem into the robust problem of networked control system. According to the established Markov jump system model and stability analysis, a robust predictive controller for networked control systems is designed by using linear matrix inequality (LMI) method, which makes the system asymptotically stable. Finally, a numerical example is given to verify the effectiveness of the proposed control method.Networked control system; medium access constraints; packet loss; robust predictive controller.
\end{abstract}

\section{Introduction}

Networked control system is more and more widely used in our real life. It is a closed-loop control system formed by sensors, controllers and actuators through real-time network[1]. Networked control system is a new technology formed in the field of automatic control. It is a new product integrating the principles of communication, computer and automatic control. Its main components include sensors, controllers and actuators. The wide application of networked control system benefits from its many advantages. Compared with traditional control system, The networked control system reduces the system wiring, can share information in real time, greatly improves the reliability of the system, and can also carry out remote control[2,3]. Because the network communication is carried out in data packets, the data packets can not only contain the data information of the current time, but also the data information of the past and the future, This is what the traditional control system can not do. Networked control system has penetrated into automation, microgrid, computer and communication and principle, and solved many problems that are difficult to be solved by traditional control system. Moreover, in the actual production, many key problems have been solved, and the productivity level of the enterprise has been greatly improved. It is widely used in power system, urban rail transit, industrial heating furnace, new energy equipment, cloud control system, aerospace, industrial automation, transportation and so on. While providing 
convenience, networked control system also has some problems that can not be ignored. The problems of media access constraints and packet loss in networked control system are also very common.

Media access constraints have a great impact on system stability. The so-called media access constraints are that because the bandwidth of each network is limited, at any time, only a few sensors and actuators will obtain channel access rights for data transmission and exchange[4]. People have conducted in-depth research on the problems caused by media access constraints. The authors of [5] studies the linear steady network control problem with medium access constraints. The system is modeled as a switched system, and then the sufficient conditions for the stability of the system are given on the basis of dwell time by using Lyapunov function method. The authors of [6] studies the minimum data rate problem of networked control systems under media access constraints. In order to make the system stable, a new analysis method of unstable scalar and vector is established, and finally the necessary and sufficient conditions for system stability are obtained. The authors of [7] studies the second-order multi-agent formation problem of wireless networks under media access constraints. By designing the agent node scheduling protocol, the qualified agent nodes can transmit information through the network. The authors of [8] studies the event triggered collaborative design method of nonlinear networked control systems with medium access constraints. On the basis of considering nonlinear dynamics, an event triggered scheme with adjustable trigger conditions is proposed. For networked control systems with media access constraints, different analysis methods are used to make the system asymptotically stable, but only a single media access constraint problem is considered in the above literature, which is not practical in practice.

Packet loss is also one of the common problems in networked control system. Packet loss is a phenomenon that the transmitted data cannot reach or reach the next node in time based on the reliability of the network itself and the influence of the network environment[9]. For some control systems with robust performance, although a certain probability of packet loss can be allowed, it will also have a certain impact on the system performance, which may lead to system instability. The impact of packet loss on system performance is also an important factor worthy of attention. Under this background, many scholars have conducted in-depth research on packet loss. Aiming at the problem of random packet loss, literature [10] estimates the state of networked control system, and eliminates various effects of packet loss on system state estimation by solving linear matrix. The authors of [11] studies the simultaneous estimation of equipment state and packet loss at each time step. After solving the problem, two solutions are proposed. Finally, an example is given to verify the effectiveness of the proposed method. The authors of [12] studies the problem of fault detection in nonlinear networked control systems. Assuming that data packets are lost during data transmission, it is modeled as a white noise sequence with Bernoulli distribution. The authors of [13] studies the dynamic output feedback fuzzy control problem triggered by adaptive events. The data packet loss is described by Bernoulli random process, and a fuzzy dynamic output feedback controller is designed to ensure the stability of the system. The above literature designs the controller through different methods for the networked control system with packet loss, so that the system can reach an asymptotically stable state in case of network packet loss. However, in reality, media access constraints and packet loss usually exist at the same time. The above control method for a single network problem is relatively conservative. Now most research work is based on an ideal assumption, that is, the system noise and process noise meet the white noise of Bernoulli distribution. In the actual industrial process, the noise is not the white noise that simply meets the characteristics of Bernoulli distribution, but some information sources with limited energy. If we ignore these practical useful information, it will inevitably reduce the estimation accuracy and estimation performance. 
However, most literatures only consider the influence of a single factor, which makes the system performance not optimal. In this paper, considering the influence of network factors such as medium access constraints and packet loss, the network problem is transformed into the robustness problem of networked control system by using Markov jump rule and Bernoulli independent identically distributed process. According to the established Markov jump system model and stability analysis, a robust predictive controller for networked control system is designed by using linear matrix inequality method, so that the system can reach asymptotic stability.

\section{Model establishment}

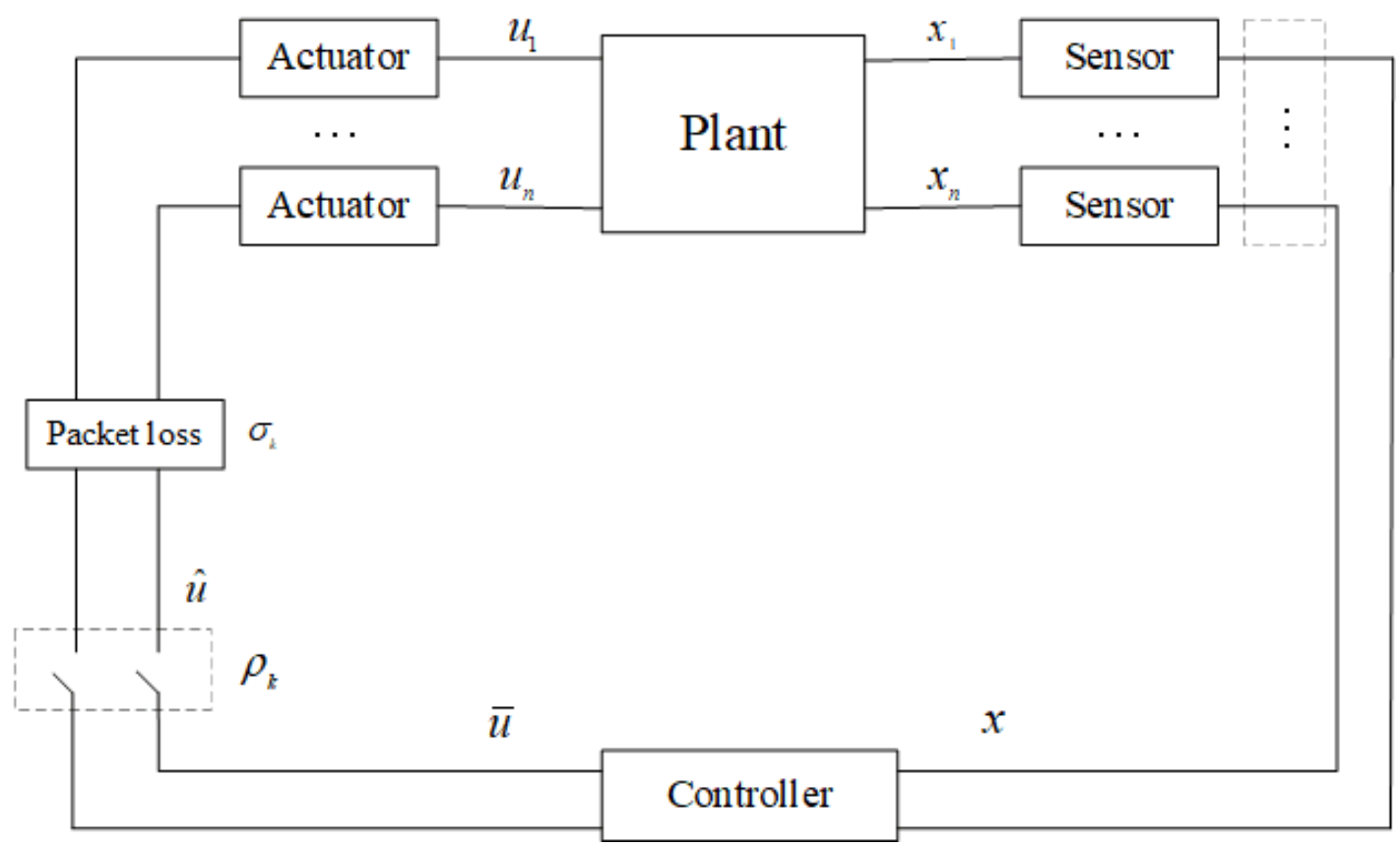

Fig. 1 The structure of networked control systems

Considering the NCS shown in Figure 1, the linear discrete system model of the controlled object is

$$
x(k+1)=A x(k)+B u(k)+D \omega(k)
$$

where $x \in R^{n}$ is the state vector; $u \in R^{m}$ is the input vectors, respectively, $A, B, D$ representing constant matrices of appropriate dimensions. In addition, it is assumed that the system noise is not white noise, but regarded as an unknown bounded deterministic variable.

The NCS in Figure 1 has $m$ actuators and $n$ sensors. Information is exchanged between the controller and the actuator through the network, and there are no communication constraints between the controller and the sensor. Due to the limitation of network bandwidth, only $p(1 \leq p<m)$ actuator can obtain the communication channel in each sampling period and execute the control command transmitted by the controller, therefore, there are $N_{m}=C_{m}^{p}$ media access modes for the actuator end of NCS. In addition, control packet loss may occur randomly during transmission due to node failure, network congestion or communication noise.

In order to describe the media access state of the actuator, the following binary functions are defined $\rho_{i}(k)$ : 


$$
\rho_{i}(k)=\left\{\begin{array}{ll}
0, & u_{i}(k)=0 \\
1, & u_{i}(k)=\bar{u}_{i}(k)
\end{array} \quad(i=1, \cdots, m)\right.
$$

where $u_{i}(k)=0$ indicates that $u_{i}(k)$ has not obtained the communication channel; $u_{i}(k)=\bar{u}_{i}(k)$ indicates that $u_{i}(k)$ has obtained the communication channel. Then the channel access state of the actuator at time $k$ can be represented by the communication sequence $\rho(k)=\left[\rho_{1}(k), \rho_{2}(k), \cdots, \rho_{m}(k)\right]^{T}$. Note, $M_{\rho}(k)=\operatorname{diag}\{\rho(k)\}$, then the input of the controlled object is

$$
\hat{u}(k)=M_{\rho}(k) \bar{u}(k)
$$

Assuming that the switching between two adjacent sampling periods $M_{\rho}(k)$ follows a Markov random process, and $M_{\rho}(k)$ is taken from the finite set $\left\{M_{\rho}^{i}\right\}_{i=1}^{N}$ to meet the following conditional probability:

$$
p_{r}\left\{M_{\rho}(k+1)=M_{\rho}^{j} \mid M_{\rho}(k)=M_{\rho}^{i}\right\}=\pi_{i j}, p_{r}\left\{M_{\rho}(k)=M_{\rho}^{i}\right\}=\pi_{i}(k)
$$

where $\pi_{i j}>0$ represents $M_{\rho}(k)$ from $i$ mode to $j$ mode, $i, j \in N, \sum_{j=1}^{N} \pi_{i j}=1 \cdot \pi_{0 i}$ is the initial probability of $i$ mode. The probability distribution of each mode at time $k$ is $\pi(k)=\left[\begin{array}{llll}\pi_{1}(k) & \pi_{2}(k) & \cdots & \pi_{N}(k)\end{array}\right]^{T}$ and $\pi(k+1)=\pi^{T} \pi(k)$.

it is assumed that the control rate of the system is given by state feedback

$$
\bar{u}(k)=K(k) x(k)
$$

For the packet loss problem, we introduce a binary random variable $\sigma_{i}(k), i=1, \cdots, m$ to represent the media access state of the packet loss process at time $k$. When the packet is successfully transmitted to the actuator $\sigma_{i}(k)=1$, when the packet is lost during transmission $\sigma_{i}(k)=0$. It is assumed that for the case of any packet loss, it conforms to the Bernoulli random process of independent and identically distributed, and has the following probability distribution:

$$
\operatorname{p}_{r}\left\{\sigma_{i}(k)=1\right\}=E\left\{\sigma_{i}(k)\right\}=\bar{\sigma}_{i}, p_{r}\left\{\sigma_{i}(k)=0\right\}=1-E\left\{\sigma_{i}(k)\right\}=1-\bar{\sigma}_{i}
$$

where $\bar{\sigma}_{i} \in[0,1]$ is a known constant, which represents the arrival probability of the $i$ channel packet of the control input. Considering the impact of packet loss, the final control signal received from the actuator can be expressed as follows:

$$
u(k)=N_{\sigma}(k) \hat{u}(k)
$$

where $N_{\sigma}(k)=\operatorname{diag}\left\{\sigma_{1}(k), \sigma_{2}(k), \cdots, \sigma_{m}(k)\right\} \quad$ represents the probability matrix of successful transmission of control signal.

Based on equations (1), (2) and (6), the discrete model of networked control system can be described as

$$
x(k+1)=A x(k)+B N_{\sigma}(k) M_{\rho}(k) \bar{u}(k)+D \omega(k)
$$

\section{Main results}

\subsection{Stability analysis}

Before the analysis of the main results, the following lemmas are given: 
Lemma1 14]: Assumed symmetric matrix $S=\left[\begin{array}{ll}S_{11} & S_{12} \\ S_{21} & S_{22}\end{array}\right]$, where $S_{11} \in R^{r \times r}$, then the following conditions are equivalent:

(1) $S<0$.

(2) $S_{22}<0, S_{11}-S_{12} S_{22}^{-1} S_{12}^{T}<0$.

(3) $S_{11}<0, S_{22}-S_{12}^{T} S_{11}^{-1} S_{12}<0$.

Lemma2 ${ }^{[14]}: H, E$ is a real matrix with appropriate dimension, $F$ is a positive definite symmetric matrix and satisfies $F^{T} F \leq I$, then there is a scalar $\varepsilon>0$ such that the following inequality holds:

$$
H F E+E^{T} F^{T} H^{T} \leq \varepsilon H H^{T}+\varepsilon^{-1} E^{T} E
$$

As we all know, model predictive control has the advantages of good control effect and strong robustness. It can deal with various constraints in controlled variables and operating variables. Firstly, the following prediction model is given:

$$
\begin{aligned}
x(k+l+1 \mid k) & =A x(k+l \mid k)+B u(k+l \mid k) \\
& =A x(k+l \mid k)+B N_{\sigma}(k+l) M_{\rho}(k+l) \bar{u}(k+l \mid k), l \geq 0
\end{aligned}
$$

where $x(k+l \mid k)$ represents the system state of predicting the $k+l$ time based on the known information of $k$ time, and $\bar{u}(k+l \mid k)$ represents the controller output of predicting the $k+l$ time based on the known information of $k$ time, then the prediction output sequence $U_{0}^{\infty}$ of the controller can be expressed by the following formula:

$$
U_{0}^{\infty}(k)=\left[u(k \mid k), \quad U_{1}^{\infty}(k)\right], U_{1}^{\infty}(k)=\left\{u(k+l \mid k) \in R^{m}: u(k+l \mid k)=K(k) x(k+l \mid k), l \geq 1\right\}
$$

Among them, $u(k \mid k)$ is the first element in the prediction output sequence $U_{0}^{\infty}$ and satisfies $u(k)=u(k \mid k)$, the prediction output $U_{0}^{\infty}$ can be calculated by the feedback control law $u(k+l \mid k)(l \geq 1)$ at $k$ time. Based on the quadratic performance index $J(k)$ in infinite time domain, the networked robust predictive control problem of solving the predictive output sequence $U_{0}^{\infty}$ at $k$ time can be transformed into the following online optimization problem:

$$
\begin{gathered}
\min _{U_{0}^{\infty}} \max J(k)=\min _{U_{0}^{\infty}} \max \sum_{l=0}^{\infty}\left[\|x(k+l \mid k)\|_{Q}^{2}+\left\|N_{\sigma}(k+l) M_{\rho}(k+l) \bar{u}(k+l \mid k)\right\|_{R}^{2}\right] \\
=\min _{U_{0}^{\infty}} \max \left[J_{0}(k)+J_{1}(k)\right] \\
E\left[J_{0}(k)\right]=E\left[x^{T}(k \mid k) Q x(k \mid k)+\left[N_{\sigma}(k) M_{\rho}(k) \bar{u}(k \mid k)\right]^{T} R\left[N_{\sigma}(k) M_{\rho}(k) \bar{u}(k \mid k)\right]\right] \\
E\left[J_{1}(k)\right]=E\left[\sum _ { l = 0 } ^ { \infty } \left\{x^{T}(k+l \mid k) Q x(k+l \mid k)+\right.\right. \\
\left.\left.\left[N_{\sigma}(k+l) M_{\rho}(k+l) \bar{u}(k+l \mid k)\right]^{T} R\left[N_{\sigma}(k+l) M_{\rho}(k+l) \bar{u}(k+l \mid k)\right]\right\}\right]
\end{gathered}
$$

where the symmetry matrix $Q=Q^{T}>0, R=R^{T}>0$ are the state and input weighting matrix, $u(k \mid k)$ is the optimization variable in the objective function. Assuming that the state $x(k)=x(k \mid k)$ of the system is measurable at $k$ time, the control goal is to adjust the initial state $x(0)$ of the system to the origin.

The above optimization problem is Min-Max optimization problem with infinite optimization variables, a linear state feedback control strategy $u(k+l \mid k)=K(k) x(k+l \mid k), l \geq 1$ is used to obtain the 
predictive output of the controller. In order to simplify the optimization problem and ensure the asymptotic stability of the system, an upper bound of the performance index $J(k)$ and a mandatory inequality constraint are constructed. First, define the quadratic function as follows:

$$
V(x(k+l \mid k))=x^{T}(k+l \mid k) P\left(s_{k}\right) x(k+l \mid k), P>0, l \geq 1
$$

Where, $\left\{s_{k}, k \in N\right\}$ is the Markov chain taking value in finite space $S=\{1,2, \cdots, L\}$. The transition probability from the $i$ mode to the $j$ mode at $k$ time is $\varsigma_{i j}=\operatorname{Pr}\left\{s_{k+1}=j \mid s_{k}=i\right\}, \varsigma_{i j} \geq 0, i, j \in S$ and $\sum_{j=1}^{L} \varsigma_{i j}=1$.

Equation (11) can be rewritten as

$$
V(x(k+l \mid k))=x^{T}(k+l \mid k) P\left(s_{k}\right) x(k+l \mid k)=x^{T}(k+l \mid k) P(i) x(k+l \mid k)
$$

the following robust stability constraints are satisfied:

$$
V(k+l+1 \mid k)-V(k+l \mid k)<-\left(\|x(k+l \mid k)\|_{Q}^{2}+\left\|N_{\sigma}(k+l) M_{\rho}(k+l) \bar{u}(k+l \mid k)\right\|_{R}^{2}\right)
$$

under the condition that the system (7) is asymptotically stable, $x(\infty \mid k)=0$ and $V(\infty \mid k)=0$, calcumlate and sum equation (13) from $l=1$ to $l=\infty$ and take the expectation

$$
\max E\left[J_{1}(k)\right]<E[V(x(k+1 \mid k))]
$$

let $\gamma(k)$ be the upper bound of $J_{0}(k)+V(x(k+1 \mid k))$, the optimization problem of robust predictive control for system (7) can be further described as:

$$
\min _{u(k \mid k), K(k), P(i)} \gamma(k)
$$

and meet the constraints (13) and mandatory constraints

$$
\begin{aligned}
& \max \left\{x^{T}(k \mid k) Q x(k \mid k)+\left[N_{\sigma}(k) M_{\rho}(k) \bar{u}(k \mid k)\right]^{T} R\left[N_{\sigma}(k) M_{\rho}(k) \bar{u}(k \mid k)\right]\right. \\
+ & \left.x^{T}(k+1 \mid k) P(i) x(k+1 \mid k)\right\}<\gamma(k)
\end{aligned}
$$

Theorem 1: Assumes that the state of networked control system (7) is measurable. For a given matrix $K(k)$ and controller output $u(k \mid k)$, if there is a well dimensioned matrix $P(i)>0, P(j)>0$ and scalar $\gamma(k)>0$, the infinite time domain performance index $J(k)$ has a minimum upper bound

$$
\min _{P(i)} \gamma(k)
$$

and the following constraints are satisfied:

$$
\left[\begin{array}{cccc}
-P(i)+Q+\delta K^{T}(k) M_{\rho}^{T}(k) N_{\sigma}^{T}(k) N_{\sigma}(k) M_{\rho}(k) K(k) & A^{T} P(j) & I & 0 \\
* & -P(j) & 0 & P(j) B \\
* & * & -R^{-1} & I \\
* & * & * & -\delta I
\end{array}\right]<0
$$




$$
\left[\begin{array}{ccccc}
-\gamma(k)+\delta \bar{u}^{T}(k \mid k) M_{\rho}^{T}(k) N_{\sigma}^{T}(k) N_{\sigma}(k) M_{\rho}(k) \bar{u}(k \mid k) & A^{T} P(j) & Q^{1 / 2} x^{T}(k \mid k) & R^{1 / 2} \bar{u}^{T}(k \mid k) & 0 \\
* & -P(j) & 0 & 0 & P(j) B \\
* & * & -I & 0 & 0 \\
* & * & * & -I & R^{1 / 2} \\
* & * & * & * & -\delta I
\end{array}\right]<0
$$

then the networked control system (7) is asymptotically stable and has certain control performance $\gamma(k)$.

Proof:Firstly, a sufficient condition for the robust stability of networked control system (7) is proved, so that inequality (18) holds. It can be obtained from equation (8), equation (12) and equation (13)

$$
\begin{array}{r}
x^{T}(k+l \mid k)\left[A+B N_{\sigma}(k) M_{\rho}(k) K(k)\right]^{T} P_{j}(k)\left[A+B N_{\sigma}(k) M_{\rho}(k) K(k)\right] x(k+l \mid k) \\
-x^{T}(k+l \mid k) P_{i}(k) x(k+l \mid k)+\left(\|x(k+l \mid k)\|_{Q}^{2}+\left\|N_{\sigma}(k) M_{\rho}(k) K(k) x(k+l \mid k)\right\|_{R}^{2}\right)<0
\end{array}
$$

inequality (21) can be obtained by applying lemma 1 to inequality (20),

$$
\left[\begin{array}{ccc}
-P(i)+Q & {\left[A+B N_{\sigma}(k) M_{\rho}(k) K(k)\right]^{T} P(j)} & K(k) M_{\rho}^{T}(k) N_{\sigma}^{T}(k) \\
* & -P(j) & 0 \\
* & * & -R^{-1}
\end{array}\right]<0
$$

inequality (21) can be decomposed into

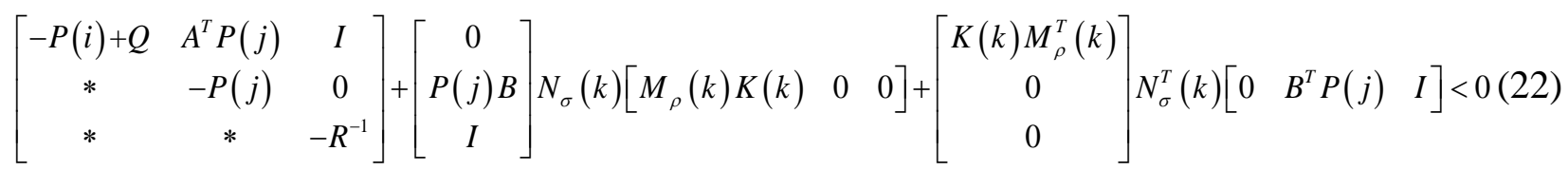

inequality (23) can be obtained by applying lemma 2 to the inequality (22)

$$
\begin{aligned}
& {\left[\begin{array}{c}
0 \\
P(j) B \\
I
\end{array}\right] N_{\sigma}(k)\left[M_{\rho}(k) K(k) \quad 0 \quad 0\right]+\left[\begin{array}{c}
K(k) M_{\rho}^{T}(k) \\
0 \\
0
\end{array}\right] N_{\sigma}^{T}(k)\left[\begin{array}{lll}
0 & B^{T} P(j) & I
\end{array}\right]} \\
& \leq \delta\left[\begin{array}{c}
K(k) M_{\rho}^{T}(k) \\
0 \\
0
\end{array}\right] N_{\sigma}^{T}(k) N_{\sigma}(k)\left[M_{\rho}(k) K(k) \quad 0 \quad 0\right]+\delta^{-1}\left[\begin{array}{c}
0 \\
P(j) B \\
I
\end{array}\right]\left[\begin{array}{lll}
0 & P(j) B & I
\end{array}\right]
\end{aligned}
$$

obviously, if the inequality (24) holds:

$\left[\begin{array}{ccc}-P(i)+Q & A^{T} P(j) & I \\ * & -P(j) & 0 \\ * & * & -R^{-1}\end{array}\right]+\delta\left[\begin{array}{c}K(k) M_{\rho}^{T}(k) \\ 0 \\ 0\end{array}\right] N_{\sigma}^{T}(k) N_{\sigma}(k)\left[M_{\rho}(k) K(k) \quad 0 \quad 0\right]+\delta^{-1}\left[\begin{array}{c}0 \\ P(j) B \\ I\end{array}\right]\left[\begin{array}{lll}0 & P(j) B & I\end{array}\right]<0(24)$

then inequality (18) can be proved by lemma 1 .

Next, it is proved that inequality (19) holds, system (7) has certain control performance, inequality (16) can be further transformed into

$$
x^{T}(k \mid k) Q x(k \mid k)+\left[N_{\sigma}(k) M_{\rho}(k) \bar{u}(k \mid k)\right]^{T} R\left[N_{\sigma}(k) M_{\rho}(k) \bar{u}(k \mid k)\right]
$$




$$
+\left[A x(k \mid k)+B N_{\sigma}(k) M_{\rho}(k) \bar{u}(k \mid k)\right]^{T} P(j)\left[A x(k \mid k)+B N_{\sigma}(k) M_{\rho}(k) \bar{u}(k \mid k)\right]<\gamma(k)
$$

inequality (26) can be obtained by applying lemma 1 to inequality (25)

$$
\begin{aligned}
& {\left[\begin{array}{cccc}
-\gamma(k) & x^{T}(k \mid k) A^{T} P(j) & Q^{1 / 2} x^{T}(k \mid k) & R^{1 / 2} \bar{u}^{T}(k \mid k) M_{\rho}^{T}(k) N_{\sigma}^{T}(k) \\
* & -P(j) & 0 & 0 \\
* & * & -I & 0 \\
* & * & * & -I
\end{array}\right]} \\
& +\left[\begin{array}{cccc}
0 & \bar{u}^{T}(k \mid k) M_{\rho}^{T}(k) N_{\sigma}^{T}(k) B^{T} P(j) & 0 & 0 \\
* & 0 & 0 & 0 \\
* & * & 0 & 0 \\
* & * & * & 0
\end{array}\right]<0 \\
& {\left[\begin{array}{cccc}
-\gamma(k) & x^{T}(k \mid k) A^{T} P(j) & Q^{1 / 2} x^{T}(k \mid k) & R^{1 / 2} \bar{u}^{T}(k \mid k) M_{\rho}^{T}(k) N_{\sigma}^{T}(k) \\
* & -P(j) & 0 & 0 \\
* & * & -I & 0 \\
* & * & * & -I
\end{array}\right]} \\
& +\left[\begin{array}{c}
0 \\
P(j) B \\
0 \\
0
\end{array}\right] N_{\sigma}(k)\left[M_{\rho}(k) \bar{u}(k \mid k) \quad 0 \quad 0 \quad 0\right]+\left[\begin{array}{c}
\bar{u}^{T}(k \mid k) M_{\rho}^{T}(k) \\
0 \\
0 \\
0
\end{array}\right] N_{\sigma}^{T}(k)\left[\begin{array}{llll}
0 & B^{T} P(j) & 0 & 0
\end{array}\right]<0
\end{aligned}
$$

inequality (28) can be obtained by applying lemma 2 to inequality (27)

$$
\begin{aligned}
& {\left[\begin{array}{c}
0 \\
P(j) B \\
0 \\
0
\end{array}\right] N_{\sigma}(k)\left[M_{\rho}(k) \bar{u}(k \mid k) \quad 0 \quad 0 \quad 0\right]+\left[\begin{array}{c}
\bar{u}^{T}(k \mid k) M_{\rho}^{T}(k) \\
0 \\
0 \\
0
\end{array}\right] N_{\sigma}^{T}(k)\left[\begin{array}{llll}
0 & B^{T} P(j) & 0 & 0
\end{array}\right]} \\
& \leq \delta\left[\begin{array}{c}
\bar{u}^{T}(k \mid k) M_{\rho}^{T}(k) \\
0 \\
0 \\
0
\end{array}\right] N_{\sigma}^{T}(k) N_{\sigma}(k)\left[M_{\rho}(k) \bar{u}(k \mid k) \quad 0 \quad 0 \quad 0\right]+\delta^{-1}\left[\begin{array}{c}
0 \\
P(j) B \\
0 \\
0
\end{array}\right]\left[\begin{array}{llll}
0 & B^{T} P(j) & 0 & 0
\end{array}\right]
\end{aligned}
$$

if the matrix inequality of equation (29) holds:

$$
\begin{aligned}
& {\left[\begin{array}{cccc}
-\gamma(k) & x^{T}(k \mid k) A^{T} P(j) & Q^{1 / 2} x^{T}(k \mid k) & R^{1 / 2} \bar{u}^{T}(k \mid k) M_{\rho}^{T}(k) N_{\sigma}^{T}(k) \\
* & -P(j) & 0 & 0 \\
* & * & -I & 0 \\
* & * & * & -I
\end{array}\right]} \\
& +\delta\left[\begin{array}{c}
\bar{u}^{T}(k \mid k) M_{\rho}^{T} \\
0 \\
0 \\
0
\end{array}\right] N_{\sigma}^{T}(k) N_{\sigma}(k)\left[M_{\rho}(k) \bar{u}(k \mid k) \quad 0 \quad 0 \quad 0\right]+\delta^{-1}\left[\begin{array}{c}
0 \\
P(j) B \\
0 \\
0
\end{array}\right]\left[\begin{array}{llll}
0 & B^{T} P(j) & 0 & 0
\end{array}\right]<0
\end{aligned}
$$

then the inequality (27) holds, from lemma 1, inequality (29) is equivalent to inequality (19). Proof complete. 


\subsection{Robust predictive controller design}

Theorem 2: It is assumed that the state of the networked control system (7) is measurable, if there is a matrix $X_{1}(k)>0, X_{2}(k)>0, Y(k)$, scalar $\delta>0$, controller output $\bar{u}(k \mid k)$ and scalar $\gamma(k)$ enable the performance index $J(k)$ with minimum upper bound,

$$
\min _{X_{1}(k), X_{2}(k), Y(k), \bar{u}(k \mid k)} \gamma(k)
$$

and the following constraints are satisfied:

$$
\begin{gathered}
{\left[\begin{array}{ccccc}
-X_{1}(k) & X_{1}(k) A^{T} & 0 & Y(k) M_{\rho}^{T}(k) N_{\sigma}^{T}(k) & Q^{1 / 2} X_{1}(k) \\
* & -X_{2}(k)+\delta B B^{T} & \delta B & 0 & 0 \\
* & * & -R^{-1}+\delta I & 0 & 0 \\
* & * & * & -\delta I & 0 \\
* & * & * & * & -I
\end{array}\right]<0} \\
{\left[\begin{array}{ccccc}
-\gamma(k) & x^{T}(k \mid k) A^{T} & Q^{1 / 2} X^{T}(k \mid k) & R^{1 / 2} \bar{u}^{T}(k \mid k) & \bar{u}^{T}(k \mid k) M_{\rho}^{T}(k) N_{\sigma}^{T}(k) \\
* & -X_{2}(k)+\delta B B^{T} & 0 & 0 & 0 \\
* & * & -I & 0 & 0 \\
* & * & * & -I & 0 \\
* & * & * & * & -\delta I
\end{array}\right]<0}
\end{gathered}
$$

then the networked control system (7) is asymptotically stable and has a given control performance $\gamma(k)$, control gain $K(k)$ can be obtained from the following formula:

$$
K(k)=Y(k) X_{1}^{-1}(k), P(i)=X_{1}^{-1}(k), P(j)=X_{2}^{-1}(k)
$$

Proof: Firstly, a sufficient condition for the robust control performance of networked control systems is proved, the establishment of matrix inequality (31), let $P(i)=X_{1}^{-1}(k), P(j)=X_{2}^{-1}(k)$, the matrix inequality (20) can be obtained by applying lemma 1

$$
\left[\begin{array}{ccc}
-P(i)+Q & {\left[A+B N_{\sigma}(k) M_{\rho}(k) K(k)\right]^{T}} & K(k) M_{\rho}^{T}(k) N_{\sigma}^{T}(k) \\
* & -P^{-1}(j) & 0 \\
* & * & -R^{-1}
\end{array}\right]<0
$$

where $P(i), P(j)$ is a nonsingular matrix. Contract transformation is performed on equation (34), that is, the left and right sides are multiplied by the diagonal matrix at the same time $\operatorname{diag}\left\{P^{-1}(i), I, I\right\}$, inequality (35) can be obtained by combining formula (33)

$$
\left[\begin{array}{ccc}
-X_{1}(k)+X_{1}(k) Q X_{1}(k) & {\left[A X_{1}(k)+B N_{\sigma}(k) M_{\rho}(k) Y(k)\right]^{T}} & Y(k) M_{\rho}^{T}(k) N_{\sigma}^{T}(k) \\
* & -X_{2}(k) & 0 \\
* & * & -R^{-1}
\end{array}\right]<0
$$

inequality (35) can be rewritten as 


$$
\begin{aligned}
& {\left[\begin{array}{ccc}
-X_{1}(k)+X_{1}(k) Q X_{1}(k) & X_{1}(k) A^{T} & 0 \\
* & -X_{2}(k) & 0 \\
* & * & -R^{-1}
\end{array}\right]+} \\
& {\left[\begin{array}{c}
0 \\
B \\
I
\end{array}\right] N_{\sigma}(k)\left[M_{\rho}(k) Y(k) \quad 0 \quad 0\right]+\left[\begin{array}{c}
Y(k) M_{\rho}^{T}(k) \\
0 \\
0
\end{array}\right] N_{\sigma}^{T}(k)\left[\begin{array}{lll}
0 & B^{T} & I
\end{array}\right]<0}
\end{aligned}
$$

inequality (37) can be obtained by applying lemma 2 to the left of inequality (36)

$$
\begin{aligned}
& {\left[\begin{array}{c}
0 \\
B \\
I
\end{array}\right] N_{\sigma}(k)\left[M_{\rho}(k) Y(k) \quad 0 \quad 0\right]+\left[\begin{array}{c}
Y(k) M_{\rho}^{T}(k) \\
0 \\
0
\end{array}\right] N_{\sigma}^{T}(k)\left[\begin{array}{lll}
0 & B^{T} & I
\end{array}\right]} \\
& \leq \delta\left[\begin{array}{c}
0 \\
B \\
I
\end{array}\right]\left[\begin{array}{lll}
0 & B^{T} & I
\end{array}\right]+\delta^{-1}\left[\begin{array}{c}
Y(k) M_{\rho}^{T}(k) \\
0 \\
0
\end{array}\right] N_{\sigma}^{T}(k) N_{\sigma}(k)\left[M_{\rho}(k) Y(k) \quad 0 \quad 0\right]
\end{aligned}
$$

if the inequality (37) holds:

$$
\left[\begin{array}{ccc}
-X_{1}(k)+X_{1}(k) Q X_{1}(k) & X_{1}(k) A^{T} & 0 \\
* & -X_{2}(k) & 0 \\
* & * & -R^{-1}
\end{array}\right]+\delta\left[\begin{array}{c}
0 \\
B \\
I
\end{array}\right]\left[\begin{array}{ccc}
0 & B^{T} & I
\end{array}\right]+\delta^{-1}\left[\begin{array}{c}
Y(k) M_{\rho}^{T}(k) \\
0 \\
0
\end{array}\right] N_{\sigma}^{T}(k) N_{\sigma}(k)\left[M_{\rho}(k) Y(k) \quad 0 \quad 0\right]<0
$$

then inequality (36) holds, from lemma 1, inequality (38) is equivalent to inequality (31).

Next, it is proved that matrix inequality (32) holds, it can be obtained from equation (25) and lemma 1

$$
\left[\begin{array}{cccc}
-\gamma(k) & x^{T}(k \mid k) A^{T} & Q^{1 / 2} x^{T}(k \mid k) & R^{1 / 2} \bar{u}^{T}(k \mid k) \\
* & -X_{2}(k) & 0 & 0 \\
* & * & -I & 0 \\
* & * & * & -I
\end{array}\right]+\left[\begin{array}{cccc}
0 & \bar{u}^{T}(k \mid k) M_{\rho}^{T}(k) N_{\sigma}^{T}(k) B^{T} & 0 & 0 \\
* & 0 & 0 & 0 \\
* & * & 0 & 0 \\
* & * & * & 0
\end{array}\right]<0
$$

inequality (39) can be rewritten as

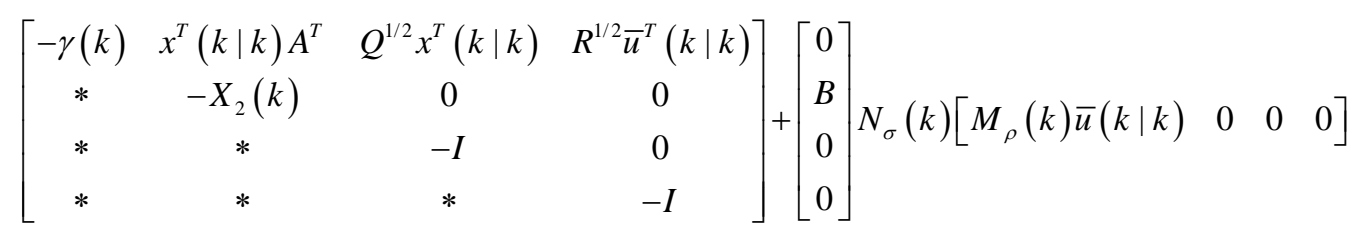

$$
\begin{aligned}
& +\left[\begin{array}{c}
\bar{u}^{T}(k \mid k) M_{\rho}^{T}(k) \\
0 \\
0 \\
0
\end{array}\right] N_{\sigma}^{T}(k)\left[\begin{array}{llll}
0 & B^{T} & 0 & 0
\end{array}\right]<0
\end{aligned}
$$

inequality (41) can be obtained by applying lemma 2 to the inequality (40)

$$
\left[\begin{array}{l}
0 \\
B \\
0 \\
0
\end{array}\right] N_{\sigma}(k)\left[M_{\rho}(k) \bar{u}(k \mid k) \quad 0 \quad 0 \quad 0\right]+\left[\begin{array}{c}
\bar{u}^{T}(k \mid k) M_{\rho}^{T}(k) \\
0 \\
0 \\
0
\end{array}\right] N_{\sigma}^{T}(k)\left[\begin{array}{llll}
0 & B^{T} & 0 & 0
\end{array}\right]
$$




$$
\leq \delta\left[\begin{array}{c}
0 \\
B \\
0 \\
0
\end{array}\right]\left[\begin{array}{llll}
0 & B^{T} & 0 & 0
\end{array}\right]+\delta^{-1}\left[\begin{array}{c}
\bar{u}^{T}(k \mid k) M_{\rho}^{T}(k) \\
0 \\
0 \\
0
\end{array}\right] N_{\sigma}^{T}(k) N_{\sigma}(k)\left[M_{\rho}(k) \bar{u}(k \mid k) \quad 0 \quad 0 \quad 0\right]
$$

if the inequality (42) holds:

$$
\begin{aligned}
& {\left[\begin{array}{cccc}
-\gamma(k) & x^{T}(k \mid k) A^{T} & Q^{1 / 2} x^{T}(k \mid k) & R^{1 / 2} \bar{u}^{T}(k \mid k) \\
* & -X_{2}(k) & 0 & 0 \\
* & * & -I & 0 \\
* & * & * & -I
\end{array}\right]} \\
& +\delta\left[\begin{array}{c}
0 \\
B \\
0 \\
0
\end{array}\right]\left[\begin{array}{llll}
0 & B^{T} & 0 & 0
\end{array}\right]+\delta^{-1}\left[\begin{array}{c}
\bar{u}^{T}(k \mid k) M_{\rho}^{T}(k) \\
0 \\
0 \\
0
\end{array}\right] N_{\sigma}^{T}(k) N_{\sigma}(k)\left[M_{\rho}(k) \bar{u}(k \mid k) \quad 0 \quad 0 \quad 0\right]<0
\end{aligned}
$$

then inequality (40) holds, by applying lemma 1 to the above inequality, inequality (42) is equivalent to inequality (32). Proof complete.

\section{Simulation example}

Consider the following open-loop unstable linear discrete-time controlled objects:

$$
A=\left[\begin{array}{cc}
0.2 & -0.5 \\
0.3 & 0.5
\end{array}\right], B=\left[\begin{array}{cc}
0.5 & 0.1 \\
1.6 & 0.3
\end{array}\right], D=\left[\begin{array}{cc}
0.15 & 0.32 \\
0.14 & 0.24
\end{array}\right]
$$

Select the initial state of the system is $x(0)=\left[\begin{array}{ll}5 & -3\end{array}\right]$, weighting matrix $Q=0.01$ and $R=0.01$, suppose at any time $k$, only one communication channel exists between the controller and the actuator, and the corresponding communication scheduling matrix is

$$
\left\{M_{1}, M_{2}\right\}=\left\{\left[\begin{array}{ll}
1 & 0 \\
0 & 0
\end{array}\right],\left[\begin{array}{ll}
0 & 0 \\
0 & 1
\end{array}\right]\right\}
$$

due to packet loss in networked control system, it is assumed that the probability of successful packet transmission between controller and actuator is 0.85, and the Markov state transition probability is

$$
P(i)=\left[\begin{array}{ll}
0.7 & 0.3 \\
0.5 & 0.5
\end{array}\right]
$$

according to Theorem 3, we can find that the controller gain of the system is

$$
K_{1}=\left[\begin{array}{ll}
0.0198 & 0.0002 \\
0.0258 & 1.6939
\end{array}\right], K_{2}=\left[\begin{array}{cc}
1.5225 & -0.0464 \\
-0.0125 & 0.0338
\end{array}\right]
$$

The goal of this paper is to design a networked robust predictive controller, so that the networked control system (7) can still ensure stability and good control performance in the presence of medium access constraints and packet loss. According to the optimization algorithm in theorem 2, the upper bound of the performance index $\gamma(k)$ of the system can be obtained, The performance index trajectory is shown in figure 2. It can be seen from figure 2 that the robust predictive controller designed in this paper can quickly converge to zero. figure 2 is the mode switching curve 
of Markov communication sequence between controller and actuator, and "1" and "2" in the ordinate represent the random matrix of communication sequence $M_{1}$ and $M_{2}$. figure.4 shows the packet loss state of the system, which "0" and "1" in the ordinate represent the packet loss state and no packet loss respectively. figure. 5 is the input signal of the controller. It can be seen from the figure that the system can quickly reach the asymptotically stable state in the 10th sampling period. Figure 6 is the state trajectory diagram of the system. It can be seen from the diagram that under given initial conditions, the controller designed in this paper can quickly converge the state of the closed-loop system (7) to an asymptotically stable state at the 10th sampling period. From the simulation results, we can see that the response fluctuation of the system is small, which shows that the networked robust predictive controller designed in this paper can make the closed-loop system quickly reach asymptotic stability and has good dynamic performance.

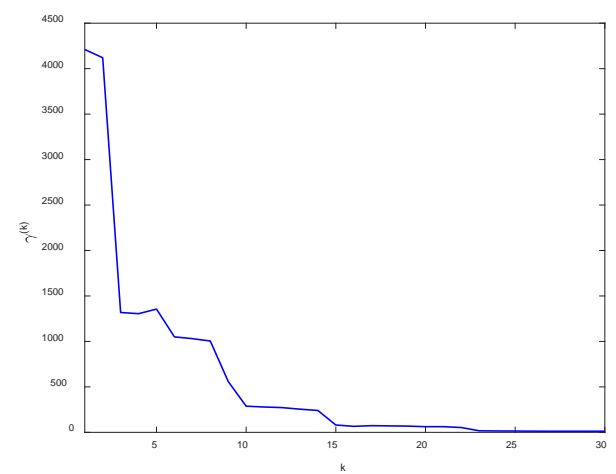

Fig.2 Track of performance $\gamma(k)$ indicators Fig.3 Switch mode of medium access sequence

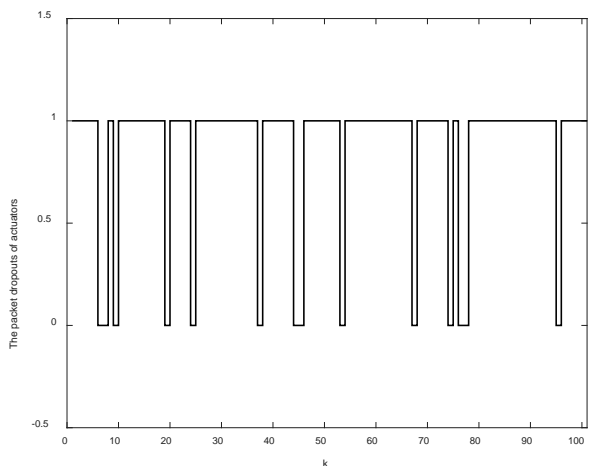

Fig.4 Actuator packet loss
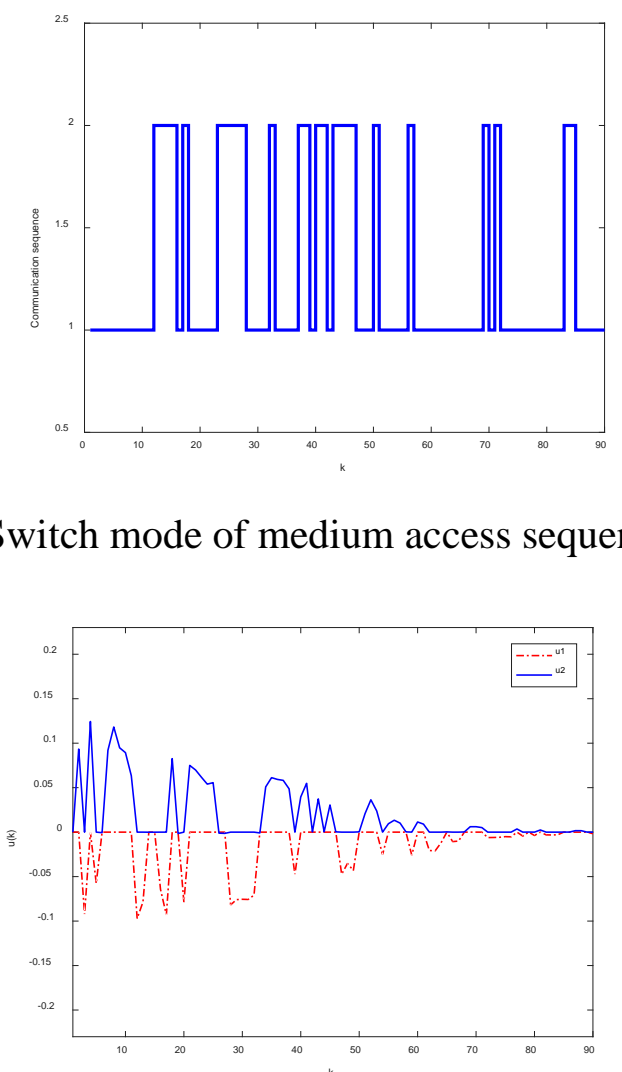

Fig. 5 Controller output signal

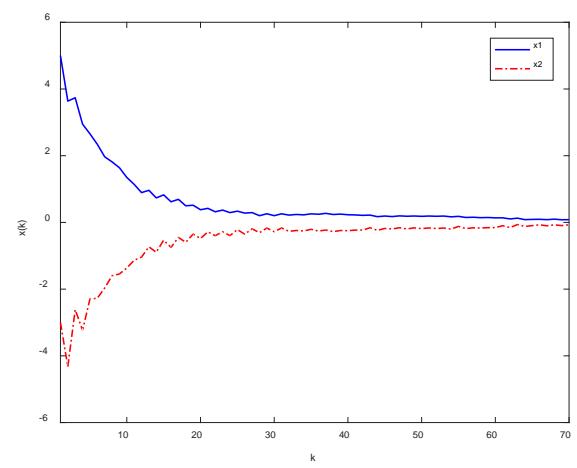

Fig. 6 State trajectory 


\section{Conclusions}

Based on the rolling time domain optimization strategy, this paper studies the networked robust predictive control with medium access constraints and packet loss. Considering the influence of network factors such as medium access constraints and packet loss, the network problem is transformed into the robustness problem of networked control system by using Markov jump rule and Bernoulli independent identically distributed process. According to the established Markov jump system model and stability analysis, a robust predictive controller for networked control system is designed by using linear matrix inequality method, so that the system can reach asymptotic stability. Finally, a numerical example is given to verify the effectiveness of the proposed control method.

\section{References}

[1] Zhang X M, Han Q L, Ge X H, et al. Networked control systems: A survey of trends and techniques[J]. IEEE/CAA Journal of Automatica Sinica, 2020, 7(1): 1-17.

[2] Wang H, Xie S S, Zhou B, et al. Non-fragile robust filtering of takagi-sugeno fuzzy networked control systems with sensor failures [J]. Sensors, 2019, 20(1).

[3] Rehak, B, Lynnyk, V. Network-based control of nonlinear large-scale systems composed of identical subsystems[J]. Journal of the Franklin Institute, 2019, 356(2): 1088-1112.

[4] Zhu C Q, Yang B, Lu C Y, et al. Robust H-infinity control for in networked control systems with communication contraints[J].Journal of Lanzhou university of technology, 2016, 42(6): 87-93.

[5] Wang L Y, Yue W. A dwell time switching approach to channel assignment for stabilization of NCSs with medium access constraint[J]. IEEE Access, 2019, 7: 107311-107319.

[6] Wen S X, Guo G. Minimum data rate for exponential stability of networked control systems with medium access constraints[J]. International Journal of Control Automation and Systems, 2018, 16(2): 717-725.

[7] Song N, Hong X M, Zhou C. Formation control of networked multi-agent systems with communication contraints [J]. Journal of dynamic and control, 2017,15(2):163-172.

[8] Wu W, Zhang Y. Event-triggered fault-tolerant control and scheduling codesign for nonlinear networked control systems with medium access constraint and packet disordering[J]. International Journal of Robust and Nonlinear Control, 2018, 28(4): 1182-1198.

[9] Zhu C Q, Yang B, Zhu X, et al. Stabilization of linear systems over networks with limited communication capacity [J]. IEEE Access, 2019, 7: 123625-123637.

[10]Zhang J F, Xue B Q. Design of state estimation of networked control systems based on rolling time domain random pack loss[J]. Electronic design engineering, 2020, 28(6):121-124.

[11] Mohammadzadeh A, Tavassoli B, Moaveni B. Simultaneous estimation of state and packet-loss occurrences in networked control systems [J]. Electrical Engineering and Systems Science, 2020.

[12] Kargar, H, Zarei, J, Razavi F. Robust fault detection filter design for nonlinear networked control systems with time-varying delays and packet dropout[J].Circuits, systems, and signal processing, 2019, 38(1):63-84.

[13] Zhang $\mathrm{Z} \mathrm{X}$, Liang $\mathrm{H} \mathrm{J}, \mathrm{Wu} C \mathrm{~W}$, et al. Adaptive event-triggered output feedback fuzzy control for nonlinear networked systems with packet dropouts and actuator failure[J]. IEEE Transactions on Fuzzy Systems, 2019, 27(9): 1793-1806.

[14] Xu S, Lam J. Robust control and filtering of singular systems[M]. Beijing: Springer Berlin Heidelberg, 2006.

[15] Xie L H. Output feedback control of systems with parameter uncertainty[J]. International Journal of Control, 1996, 63(4):741-750. 